\title{
Multidimensional Modulation and Coding
}

\author{
Magnus Karlsson (1) and Erik Agrell (2) \\ (1) Department of Microtechnology and Nanoscience, (2) Department of Signals and Systems \\ Chalmers University of Technology, SE-412 96 Göteborg, Sweden \\ email: magnus.karlsson@chalmers.se
}

\begin{abstract}
We discuss how error-correction coding and modulation can be cooptimized, how modulation formats can be interpreted as codes, and the roles of soft and hard decision decoding.
\end{abstract}

OCIS codes: (060.0060) Fiber optics and optical communications; (060.4080) Modulation; (060.4510) Optical communications

\section{Introduction}

The classical schematic of a communication link consists of, innermost, a physical channel, through which a discrete sequence of symbols (chosen from a discrete set, the modulation format) are transmitted. Each symbol may carry one or more bits of data directly, or one may use an encoder/decoder to apply a forward error correcting (FEC) code on the symbol bits. The properties of the channel and the choice of symbols are usually dictated by the physical properties of the hardware used.

For example, coherent fiber optic channels are (in the linear regime) well modeled by the additive white Gaussian noise (AWGN) channel [1,2], with a four-dimensional (4d) symbol space [2,3], composed of the two quadratures in the two polarization components of the electromagnetic field. The Mach-Zehnder modulators [4], commonly used to modulate the four quadratures, have a limited dynamic range, confining the transmitted symbols to within a $4 \mathrm{~d}$ hypercube. The receiver requires a significant amount of digital signal processing (DSP) to track the randomly varying phase and polarization of the fiber channel, and to equalize impairments, before the transmitted data can be detected.

Shannon showed that every channel has an upper limit, the channel capacity, to the maximum data rate it can carry with arbitrarily low bit-error rate [5]. In addition, for a chosen modulation format, the mutual information (MI) will provide a more restrictive limit on the maximum data rate. For a discrete-time memoryless channel, the capacity is the highest MI among all possible modulation formats. It was also shown that in order to approach these limits, the dimensionality of the data transmitted over the channel must approach infinity [6]. In practice, this is done by using the outer FEC code. The code forms sets of (in state-of the art systems comprising tens of thousands) bits to a multidimensional frame that is transmitted, which enables the MI to be approached. It follows that the MI can only be reached when the channel latency (proportional to the code frame size) approaches infinity.

There are now a number of ways to cooptimize modulation and coding to approach Shannon's limits. We will classify them depending on their receiver detection schemes, from simpler that require high signal to noise ratio (SNR), to more complex and SNR tolerant. It is instructive to make two distinctions: soft and hard decisions on symbols (which we will call demodulation) or bits (which we call decoding). Soft and hard decision differ significantly in terms of complexity and performance. We will in this context consider three cases. First, we can use no coding but an efficient multidimensional modulation format with maximum likelihood demodulation. This is useful in, e.g., systems with latency demands that do not allow for long FEC codes. A second alternative is to use hard-decision demodulation and decoding, which is a good trade-off, e.g., in direct-detection links where decoding and demodulation must be simple and affordable and consume little electronic resources. The third and most advanced scheme, with best performance, is to use soft-decision demodulation and decoding. We will briefly discuss these schemes in this contribution, which is loosely based on [7, Sec 2.4].

\section{Demodulation as soft-decision decoding}

The idea behind this scheme is to use power-efficient multidimensional modulation formats (constellations), which are optimally (in the maximum likelihood sense) demodulated by using soft data (which are the received symbol coordinates), without the use of an external FEC code. To compare formats of dimensionality $N$, with $M$ levels, we use the minimum Euclidean distance of the constellation $d_{\min }$ and the average symbol energy $E_{s}$ to form the (dimensionless) power efficiency $\gamma=d_{\min }^{2} \log _{2}(M) /\left(4 E_{s}\right)$, and the spectral efficiency $\mathrm{SE}=\log _{2}(M) /(N / 2)$ in units of bit/s/polarization [2]. For lower dimensions one can use sphere-packing algorithms to find optimum constellations 


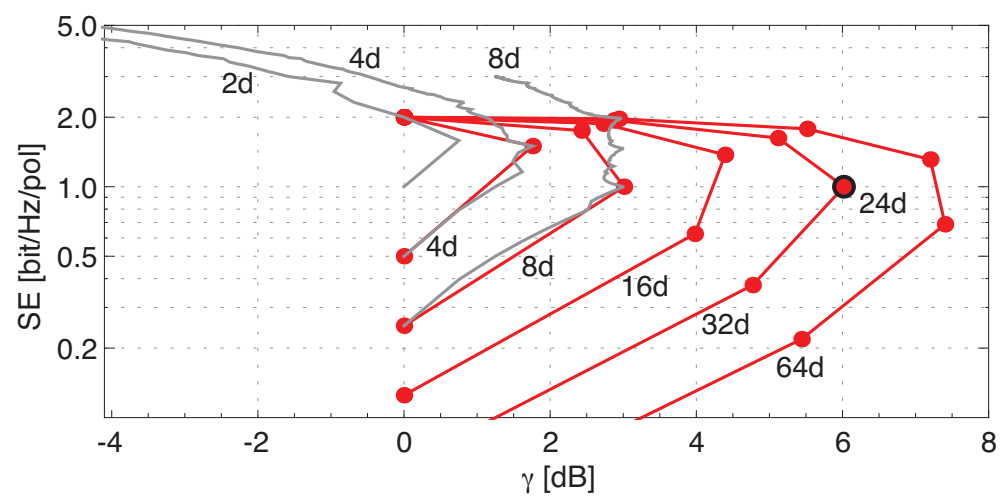

Fig. 1: Spectral efficiency vs. gamma for optimum formats in 2d, 4d, 8d (grey). The red dots show BPSK using RM(r,u)-codes for $u=2, \ldots, 6$, marked with dimensionality $2^{u}$, and as the black ring, the $24 \mathrm{~d}$ extended Golay code.

(highest $\gamma$ ) for each $M$ and $N$, and these optima are shown as grey lines in Fig. 1 for dimensions $N=2,4,8$. However, such optimizations are very demanding for higher dimensionalities, and the obtained constellations are often intractable for practical deployment, requiring large memory and processing resources for storage and demodulation. Alternatively, good constellations for higher dimensions can be found based on known results from coding theory in the following way: Use a simple modulation format together with a code with good distance properties. Similar ideas have been used in the communication literature since the sixties [8,9], and more recently within optical transmission [10].

To give a few examples, consider the binary block code $\left(n, k, d_{\mathrm{H}}\right)$, where $n$ is the code length (dimensionality), $k$ is the number of information bits and $d_{\mathrm{H}}$ is the minimum (Hamming) distance of the code. The code rate is $R=k / n$, which is often equivalently expressed in terms of the overhead $1 / R-1$. We will now use this code together with simple binary phase shift keying (BPSK) modulation, and this will result in a modulation format with dimensionality $N=n$, with $M=2^{k}$ points, a minimum squared Euclidean distance of $d_{\mathrm{min}}^{2}=2 d_{\mathrm{H}}$, and an energy per symbol of $E_{s}=n$. This gives $\gamma=d_{\mathrm{H}} R$ and $\mathrm{SE}=2 R$. As $R<1$, this approach will limit the $\mathrm{SE}$ to be $<2$, although higher spectral efficiencies can be obtained by starting from a format with higher SE than BPSK.

Particularly useful codes in this context are the Reed-Muller, $\operatorname{RM}(r, u)$ codes, characterised by the two integers $r, u$ chosen so that $0 \leq r \leq u$. The $\operatorname{RM}(r, u)$ has $\left(n, k, d_{\mathrm{H}}\right)=\left(2^{u}, \sum_{i=0}^{r}\left(\begin{array}{c}u \\ i\end{array}\right), 2^{u-r}\right)$. Special cases include uncoded transmission $(r=u)$, single-parity-check codes $(r=u-1)$, biorthogonal codes $(r=1)$, and repetition codes $(r=0)$. The cases $r=0, \ldots, u$ are shown connected with red lines for $u=2,3,4,5,6$ in Fig. 1 . The dimensionality of the formats is $2^{u}$, which increases from 4 to 64 as shown. We may note that the best known constellations in $4 \mathrm{~d}$ and $8 \mathrm{~d}$ are obtained by the biorthogonal codes, i.e. $\mathrm{RM}(1, u)$, for $u=2,3$. Specifically, the $\mathrm{RM}(1,2)$ code has parameters $\left(n, k, d_{\mathrm{H}}\right)=(4,3,2)$, and is known as polarization-switched quadrature phase-shift keying (PS-QPSK) [2,11]. The RM $(1,3) \operatorname{code}$ has $\left(n, k, d_{\mathrm{H}}\right)=$ $(8,4,4)$, and was implemented by Eriksson et al. [12] and in a modified form by Shiner et al. [13]. Other examples we may consider is the $24 \mathrm{~d}$ extended Golay code wth parameters $\left(n, k, d_{\mathrm{H}}\right)=(24,12,8)$, which is a particularly efficient sphere packing in 24d, investigated by Millar et al. [14] and shown as a black circle at SE $=1$ and $\gamma=6.02 \mathrm{~dB}$. It has the same performance as the RM(5,2) code which is (less efficiently) embedded in $32 \mathrm{~d}$.

This illustrates the insight that modulation formats can be interpreted as an outer code on a simpler inner format, and maximum likelihood demodulation is equivalent to soft-decision decoding of that code. Practical benefits are low latency and relatively simple implementations, provided one has access to the required number of dimensions and do not use too complicated codes. In future spatial-division multiplexed links with many parallel channels this may very well be realized [15]. However, practical implementations will likely limit dimensionalities and the achievable performance gains. The fundamental limit for this scheme is set by the mutual information (MI) of the underlying modulation format (in the above case this is BPSK), and the longer the outer code, i.e., the higher the dimensionality of the resulting format, the closer will one approach the MI of BPSK.

\section{Hard-decision decoding}

If we instead consider hard-decision symbol demodulation together with hard-decision FEC decoding, we can obtain relatively good performance with reasonable complexity. Hard-decision decoding can be implemented by using binary logic gates, and this will enable the use of stronger codes such as Reed-Solomon (RS) or Bose-ChaudhuriHoquenghem $(\mathrm{BCH})$ codes. Besides being implementable with relatively low complexity, these codes are algebraically well-structured in the sense that theory and algorithms for decoding, distance properties, and post-decoder error rate 
calculations are available, with no error floors or other nasty surprises that can happen for iteratively decoded codes such as turbo or low-density parity-check (LDPC) codes. They can also be used together with more power-efficient modulation formats such as the ones described in the previous section, albeit with the asymptotic power gains after decoding being smaller than the uncoded differences. Hard-decision decoding usually has $2-3 \mathrm{~dB}$ performance penalty over soft-decision decoding of the same code. To approach the MI of a given modulation format, one usually has to resort to soft-decision decoding. Also, the tendency to focus on pre-FEC BER (the "FEC limit") in those systems, makes optimization of the code rate more difficult, or even neglected in practice.

\section{Soft decision demodulation and decoding}

The use of more modern codes such as LDPC and turbo codes require the use of soft data in the FEC decoding. This is realized via iterative decoding schemes where the data is processed through the decoder repeatedly. At the price of increased complexity, latency and power consumption, this enables a detector that comes very close to the MI, while still being reasonable to implement. There are a number of ways that the mapping between symbols and bits can be performed; for example the MI for each bit in the used modulation format can be used to judge which bits need most coding protection, and the used codes can be optimized after that choice [16]. Simpler (but less optimal) is to apply the same code to all bits, possibly after interleaving, which is known as bit-interleaved coded modulation (BICM), studied in an optical context in [17]. Recent research has shown [18] that for BICM with practical bit-wise decoders, e.g., iterative soft-decision LDPC decoders, the use of lower-dimensionality parallel modulation schemes (like PM-QPSK) will perform well, whereas the more complex multidimensional formats (as the ones described in Sec. 2 above) are beneficial only if ideal ML detection can be applied in the decoding process. Such detection schemes are likely too complicated for today's systems, however.

\section{Summary and conclusions}

We discussed the work on coding and modulation for optical links in three cases with increasing complexity and performance; uncoded with power-efficient multidimensional modulation formats and coded with hard- and iterative soft-decision decoding. We showed how power-efficient formats can be designed by applying a (relatively) lowdimensional code to parallel channels of a simple modulation such as BPSK. The resulting multidimensional format can gain several dBs of sensitivity. Even if such a format can be used together with an outer FEC code, the resulting system with two concatenated codes would likely be suboptimal. Thus formats and codes should be jointly designed to reach the best trade-off between performance and complexity.

\section{References}

1. K. Kikuchi and S. Tsukamoto, "Evaluation of sensitivity of the digital coherent receiver", J. Lightwave Tech. 26, pp. 1817-1822 (2008).

2. E. Agrell and M. Karlsson, "Power-efficient modulation mormats in moherent transmission systems", J. Lightwave Tech. 27, pp. 5115-5126 (2009).

3. S. Betti et al., "A novel multilevel coherent optical system: 4-quadrature signaling", J. Lightwave Tech. 9, pp. 514-523 (1991).

4. H. Sun et. al, "Real-time measurements of a $40 \mathrm{~Gb} / \mathrm{s}$ coherent system", Opt. Expr. 16, pp. 873-879 (2008).

5. C. E. Shannon, "A mathematical theory of communication", Bell Syst. Tech. J. 27, pp. 379-423, 623-656 (1948).

6. C. E. Shannon, "Probability of error for optimal codes in a Gaussian channel”, Bell Syst. Tech. J. 38, pp. 611-656 (1959).

7. M. Karlsson and E. Agrell, "Multidimensional optimized modulation formats", Ch. 2 in "Enabling Technologies for High Spectral-efficiency Coherent Optical Communication Networks," ed. X. Zhou and C. Xie, Wiley 2016.

8. A. J. Viterbi, "On coded phase-coherent communications", IRE Trans. Space El. and Telem. SET-7 pp. 3-14, (1961).

9. G. D. Forney, "Multidimensional constellations-Part I: Introduction, figures of merit, and generalized cross constellations", IEEE J. Sel. Areas Commun. 7, pp. 877-892 (1989).
10. D. S. Millar et al., "High-dimensional modulation for coherent optical communications systems", Opt. Expr. 22, pp. 8798-8812 (2014).

11. M. Karlsson and E. Agrell, "Which is the most power-efficient modulation format in optical links?", Opt. Exp. 17, pp. 10814-10819 (2009).

12. T. A. Eriksson et al., "Frequency and polarization switched QPSK", in European Conference on Optical Communication, p. Th.2.D.4 (2013).

13. A. D. Shiner et al., "Demonstration of an 8-dimensional modulation format with reduced inter-channel nonlinearities in a polarization multiplexed coherent system", Opt. Exp. 22, pp. 20366-20374 (2014).

14. D. S. Millar et al., "A 24-dimensional modulation format achieving $6 \mathrm{~dB}$ asymptotic power efficiency", in Signal Processing in Photonic Communications, p. SPM3D.6 (2013).

15. B. J. Puttnam et al., "Modulation formats for multi-core fiber transmission", Opt. Exp. 22, pp. 32457-32469 (2014).

16. L. Beygi et al., "Coded modulation for fiber-optic networks: Toward better tradeoff between signal processing complexity and optical transparent reach", IEEE Sig. Proc. Mag. 31, pp. 93-103 (2014).

17. I. B. Djordjevic et al., "Using LDPC-coded modulation and coherent detection for ultra highspeed optical transmission", J. Lightwave Technol. 25, pp. 3619-3625 (2007).

18. A. Alvarado and E. Agrell, "Four dimensional coded modulation with bitwise decoders for future optical communications", J. Lightwave Technol. 33, pp. 1993-2003 (2015). 